$11-2017$

\title{
The Future Perspective: Metabolomics in Laboratory Medicine for Inborn Errors of Metabolism
}

Yana Sandlers

Cleveland State University, y.sandlers@csuohio.edu

Follow this and additional works at: https://engagedscholarship.csuohio.edu/scichem_facpub

Part of the Biochemistry Commons, and the Chemistry Commons

How does access to this work benefit you? Let us know!

Publisher's Statement

https://doi.org/10.1016/j.trsl.2017.06.005

\section{Recommended Citation}

Sandlers, Yana, "The Future Perspective: Metabolomics in Laboratory Medicine for Inborn Errors of Metabolism" (2017). Chemistry Faculty Publications. 491.

https://engagedscholarship.csuohio.edu/scichem_facpub/491

This Article is brought to you for free and open access by the Chemistry Department at EngagedScholarship@CSU. It has been accepted for inclusion in Chemistry Faculty Publications by an authorized administrator of EngagedScholarship@CSU. For more information, please contact library.es@csuohio.edu. 


\title{
The future perspective: metabolomics in laboratory medicine for inborn errors of metabolism
}

\author{
YANA SANDLERS
}

CLEVELAND, OHIO

\begin{abstract}
Metabolomics can be described as a simultaneous and comprehensive analysis of small molecules in a biological sample. Recent technological and bioinformatics advances have facilitated large-scale metabolomic studies in many areas, including inborn errors of metabolism (IEMs). Despite significant improvements in the diagnosis and treatment of some IEMs, it is still challenging to understand how genetic variation affects disease progression and susceptibility. In addition, a search for new more personalized therapies and a growing demand for tools to monitor the long-term metabolic effects of existing therapies set the stage for metabolomics integration in preclinical and clinical studies. While targeted metabolomics approach is a common practice in biochemical genetics laboratories for biochemical diagnosis and monitoring of IEMs, applications of untargeted metabolomics in the clinical laboratories are still in infancy, facing some challenges. It is however, expected in the future to dramatically change the scope and utility of the clinical laboratory playing a significant role in patient management. This review provides an overview of targeted and global, large-scale metabolomic studies applied to investigate various IEMs. We discuss an existing and prospective clinical applications of metabolomics in IEMs for better diagnosis and deep understanding of complex metabolic perturbations associated with the etiology of inherited metabolic disorders. (Translational Research 2017;189:65-75)
\end{abstract}

\begin{abstract}
Abbreviations: GC-MS = gas chromatography mass spectrometry; LC-MS = liquid chromatography mass spectrometry; $\mathrm{CSF}=$ Cerebrospinal fluid; IEM = inborn errors of metabolism; $\mathrm{MALDI}=$ matrix-assisted laser desorption ionization; SELDI = Surface-enhanced laser desorption/ionization; $\mathrm{NMR}$ = nuclear magnetic resonance; NBS = newborn screening; PKU = phenylketonuria; $\mathrm{LCHAD}=$ Long-chain 3-hydroxyacyl-CoA dehydrogenase deficiency; TFP = Trifunctional protein deficiency; $\mathrm{MMA}=$ methylmalonic acedimia; $\mathrm{PA}=$ propionic acedimia; OTC = Ornithine transcarbamylase deficiency; RDC = respiratory chain deficiencies; SLOS = Smith-Lemli Opitz syndrome; CTX = Cerebrotendinous xanthomatosis; RP = Reverse phase; HILIC = hydrophilic interaction liquid chromatography; $R O C=$ receiver-operating characteristic; $A U C=$ Area under the curve
\end{abstract}

etabolic changes as a disease symptom have already been recognized in ancient medicine. ${ }^{1}$ In the early 19th century, an English physician and pioneer of inherited metabolic disorders, Sir A. Garrod developed the concept of "chemical individuality" and suggested that there are phenotypes 
associated with specific metabolites. In the 1960s, progress in science and technology expanded our ability to test Garrod's hypothesis and study metabolism. ${ }^{2,3}$ In 1998, SG Oliver ${ }^{4}$ was the first to use the term "metabolomics," indicating comprehensive analysis of all metabolites in a biological system. Since the publication of his article, technological advances facilitating metabolomic studies have allowed the field to grow rapidly. As of April 2017, a PubMed search yields more than 15,000 published studies containing the word "metabolomics."

The metabolome can be described as the complete composition of all low molecular weight molecules present in a given biological system, fluid, cell, or tissue. $^{5}$ Endogenous metabolites indicate the end products of the complex "omics" cascade which is initiated by the genome followed by the transcriptome and proteome. Hence, the metabolome bridges the gap between a given genotype and phenotype.

\section{ANALYTICAL TOOLS TO STUDY METABOLOME}

The human metabolome is very diverse and dynamic. Physicochemical properties such as polarity and volatility, a wide dynamic range of endogenous metabolites, and the presence of exogenous metabolites obtained by diet and medication present a significant analytical challenge to the field. No single metabolomic methodology is currently able to measure the entire metabolome accurately. Depending on the goal, several metabolomics approaches and analytical platforms are available (Table I). Currently, nuclear magnetic resonance spectroscopy (NMR) and mass spectrometry (MS) are the dominant tools in the metabolomics field. While ${ }^{1} \mathrm{H}$ NMR is very common in metabolomics studies ${ }^{13} \mathrm{C}$ NMR and ${ }^{31} \mathrm{PNMR}$ are less frequent although these techniques can also be very informative. ${ }^{6,7}$ NMR relies on chemical shifts and multiplet patterns for metabolite identification. It requires minimal sample preparation with reproducible and nondestructive analysis however, it has relatively low sensitivity. Mass spectrometry is a versatile detector that provides higher sensitivity and can be implemented as separation-free (MALDI, SELDI) or combined with chromatography platforms such as GC, capillary electrophoresis or LC. In gas chromatography (GC), compounds are heated and nonvolatile metabolites require a derivatization step. Capillary electrophoresis provides excellent separation for polar and charged molecules, but suffers from low repeatability. In liquid chromatography (LC), a wide range of metabolites can be analyzed depending on the type of column and mobile phase.
Approach. Independent of the analytical technique employed, metabolite determination can be performed with two main conceptual approaches. The first, global untargeted metabolomics, is primarily data driven rather than specific hypothesis driven. This unbiased approach provides a comprehensive insight of the systemic response of the subject to the pathophysiological stimuli or genetic modifications. In the untargeted approach, data preprocessing, statistical, and pathway analyses have a very significant role. In NMR for example, to address chemical shift variability across the data, spectra are segmented into small areas called bins. The binning process significantly minimizes the effects from variations in peak positions and reduces the data size for multivariate statistical analyses. In MS, all detectable features are preprocessed through peak alignment, baseline correction, and deconvolution. The resulting preprocessed data is subject to multivariate and pattern recognition analyses such as principal component analysis, hierarchical cluster analysis, and supervised methods such as partial least squares discriminant analysis (PLS-DA) or orthogonal partial least squares discriminant analysis (OPLS-DA). Such analyses can discriminate populations with different health statuses to make predictive models and find potential biomarkers. Data preprocessing and statistical analyses are extensively reviewed by $\operatorname{Karaman}^{8}$ and Ren et $\mathrm{al}^{9}{ }^{9}$ respectively.

An additional layer of metabolomic data interpretation involves pathway analysis. Pathway analysis is a visualization of metabolic pathways and mapping groups of metabolites related to the same biological process. It is applied to metabolomic data for in-depth biological interpretation. Pathway enrichment analysis ${ }^{10}$ also identifies pathways that have significant perturbations in concentrations of involved metabolites. Pathway mapping and visualization tools are reviewed by Chagoyen et al. ${ }^{11}$

The benefit of untargeted, nonhypothesis-driven metabolomics is that it may provide unexpected and previously unknown correlations between metabolites and biochemical pathways in a systematic way. The bottleneck of this strategy however, is that most of the studies rely on complex statistical models as the endpoint of the study and do not follow up with the validation of candidate biomarkers.

In contrast to global untargeted metabolomics, metabolic profiling or targeted metabolomics, is a narrower, hypothesis-driven approach. This approach focuses on the analysis of a specific class of metabolites or group of metabolites related to a specific metabolic pathway. This type of workflow allows accurate 
Table I. Summary of the most common analytical tools in metabolomics studies

\begin{tabular}{|c|c|c|c|}
\hline $\begin{array}{l}\text { Analytical } \\
\text { platform }\end{array}$ & Principle & Advantage & Disadvantage \\
\hline NMR & $\begin{array}{l}\text { External magnetic field } \\
\text { interacts with spin active } \\
\text { nuclei }\left({ }^{1} \mathrm{H} \text { and }{ }^{13} \mathrm{C} \text { for example). }\right. \\
\text { Metabolite identification by } \\
\text { chemical shifts and multiplet patterns }\end{array}$ & $\begin{array}{l}\text { - } \text { Minimal sample preparation } \\
\text { - } \text { Nondestructive } \\
\text { - } \text { Robust } \\
\text { - Databases for identification }\end{array}$ & $\begin{array}{l}\text { - Low sensitivity } \\
\text { - High instrumentation cost }\end{array}$ \\
\hline GC-MS & $\begin{array}{l}\text { Sample is vaporized and } \\
\text { separated on column. } \\
\text { Metabolites are detected by } \\
\text { mass spectrometry }\end{array}$ & $\begin{array}{l}\text { - } \text { Reproducible } \\
\text { - } \text { High sensitivity } \\
\text { - Standard databases for } \\
\text { identification (NIST, Fiehn) }\end{array}$ & $\begin{array}{l}\text { - Polar compounds require } \\
\text { derivatization step } \\
\text { - Only volatile compounds } \\
\text { can be analyzed } \\
\text { - Low throughput } \\
\text { - Samples are not recovered }\end{array}$ \\
\hline FIA-MS & $\begin{array}{l}\text { Sample is delivered to mass } \\
\text { spectrometry with no } \\
\text { chromatographic separation }\end{array}$ & $\begin{array}{l}\text { - Very high throughput } \\
\text { - Low cost analysis } \\
\text { - Highly Automated } \\
\text { - No need for calibration } \\
\text { curve construction; } \\
\text { can be performed } \\
\text { as single-point calibration } \\
\text { - Gold standard for NBS } \\
\text { - High sensitivity }\end{array}$ & $\begin{array}{l}\text { - No separation of isobaric } \\
\text { - } \text { masses and isomeric compounds } \\
\text { ion suppression } \\
\text { - Samples are not recovered }\end{array}$ \\
\hline LC-MS/MS & $\begin{array}{l}\text { Sample is separated high } \\
\text { pressure on column. Wide } \\
\text { selection of stationary phases }\end{array}$ & $\begin{array}{l}\text { - Minimal sample preparation } \\
\text { - High-throughput capability } \\
\text { - Wide selection of stationary phases }\end{array}$ & $\begin{array}{l}\text { - Samples are not recovered } \\
\text { - Some analytes require } \\
\text { derivatization step } \\
\text { - lon suppression can } \\
\text { affect results }\end{array}$ \\
\hline $\begin{array}{l}\text { Capillary } \\
\quad \text { electrophoresis }\end{array}$ & $\begin{array}{l}\text { Ions are separated based on } \\
\text { their electrophoretic mobility } \\
\text { under applied voltage. Can be } \\
\text { coupled to mass } \\
\text { spectrometric detector }\end{array}$ & $\begin{array}{l}\text { - Very high resolution } \\
\text { - Sensitive technique for highly polar } \\
\text { and charged analytes } \\
\text { - Inorganic anions can be analyzed } \\
\text { - Can be coupled to MS }\end{array}$ & $\begin{array}{l}\text { - Samples are not recovered } \\
\text { - Low throughput }\end{array}$ \\
\hline
\end{tabular}

Abbreviations: FIA-MS, flow injection analysis-mass spectrometry; GC-MS, gas chromatography mass spectrometry; LC-MS/MS, liquid chromatography mass spectrometry; NBS, newborn screening; NMR, nuclear magnetic resonance.

identification and absolute or relative quantification of a predefined group of metabolites.

\section{INHERITED METABOLIC DISORDERS}

Inborn errors of metabolism (IEMs) represent a group of inherited conditions with a collectively estimated incidence of $1: 800 .^{12}$ The genetic basis of IEMs is very heterogeneous and can involve abnormalities such as point mutations, deletions or insertions, or more complex genomic rearrangements. Introduction of molecular genetics techniques have made it possible to identify molecular defects and confirm diagnoses in IEMs however, disease phenotypes are not always explained by the detected mutations. Indeed, the level of IEM complexity requires an integrated understanding of perturbations in genetic and biochemical networks.

Metabolically, IEMs are characterized by direct toxic accumulation of upstream metabolites or deficiency of downstream metabolites and/or channeling of excess intermediates to an alternative pathway. Any of these metabolic abnormalities can lead to morbidity and mortality. Thus, timely diagnosis and early disease management, which in many cases include life-saving dietary restrictions and nutritional supplements, are critically important. The newborn screening (NBS) program is a public health initiative that involves laboratory testing to timely diagnose even asymptomatic neonates. It is an unique system for predictive, preventive, and personalized medicine at the same time. The NBS program was launched in the United States in 1963, but it was restricted for phenylketonuria (PKU) screening. ${ }^{13}$ Since then, millions of newborn babies in the United States have been screened for genetic, endocrine, and metabolic disorders via heel-pricked blood samples collected on filter paper. ${ }^{14}$

Metabolic profiling by tandem MS for NBS was first proposed by Millington et al in $1990 .{ }^{15}$ The workflow involved quantitative analysis of a small subset of predetermined analytes extracted from dried blood spots from umbilical cord blood and neonatal blood. The platform was extended a few years later to include 
more diagnostic analytes. ${ }^{16-18}$ Currently, it is highly automated and implemented worldwide. In the United States, every newborn is routinely screened for at least 29 disorders however, recent technical advances in MS and new treatment options unlock the potential for expanding this significant public health initiative for more conditions. ${ }^{19-21}$

Despite significant improvements in the diagnosis and treatment of IEMs, it is still challenging to understand how genetic variation affects disease progression and susceptibility. In addition, a search for new personalized therapies and a growing demand for tools to monitor long-term metabolic effects of existing therapies set the stage for metabolomics integration in preclinical and clinical studies of the IEM field.

\section{APPLICATIONS OF GLOBAL METABOLOMICS IN IEMS STUDIES}

Although NBS is a well-validated and robust platform, it represents only a snapshot of a very small subset of the metabolic information. Global untargeted metabolomics has been employed by different groups in a various ways (Table II). Wikoff et al. used untargeted, large-scale MS-based metabolomics in plasma to characterize metabolic disturbances in methylmalonic acidemia (MMA) and propionic academia. As a proof of concept, propionyl carnitine (C3), a known diagnostic biomarker for both disorders, was validated through the study (ANOVA $P$ value of $\left.1.8 \times 10^{-18}\right) .{ }^{27}$ This comprehensive study also identified previously unreported plasma. However, the secondary diagnostic MMA marker, methylmalonic carnitine (C4DC), was not detected. The authors attribute this false-negative outcome to the high polarity of methylmalonic carnitine-acylcarnitine. These findings uncover the powerful potential of global metabolomics in disease pathophysiology investigations of even very well-studied disorders like MMA and propionic academia but raise a question of the analytical platform choice for the study.

To assess the global metabolic state in long-chain 3-hydroxyacyl-CoA dehydrogenase deficiency and carnitine palmitoyltransferase II deficiency-affected individuals, McCoin et al applied untargeted plasma MS-based metabolomics and detected 832 metabolites. Routinely, fatty acid oxidation disorders can be diagnosed biochemically by abnormal plasma acylcarnitines and urine organic acids however, the authors identified 114 nonacylcarnitine-discriminating metabolites including some nonlipids. ${ }^{23}$ Significantly higher levels of triglycerides and low levels of phosphatidylethanolamines, ceramides, and sphingomyelins in the plasma of affected individuals suggest
Table II. Summary of IEM metabolomics studies reviewed

\begin{tabular}{|c|c|c|}
\hline $\begin{array}{l}\text { Group/name of } \\
\text { metabolic disorders }\end{array}$ & Specifics & Citation \\
\hline $\begin{array}{l}\text { Disorders of amino } \\
\text { acids }\end{array}$ & $\begin{array}{l}\text { MSUD, PKU, } \\
\text { Citrullinemia, } \\
\text { Homocystinuria }\end{array}$ & $\begin{array}{l}\text { Chance et } \mathrm{al}^{16} \\
\text { Mütze et } \mathrm{al}^{22}\end{array}$ \\
\hline FAOD & $\begin{array}{l}\text { LCHAD, CPT2, } \\
\text { VLCAD, MCAD }\end{array}$ & 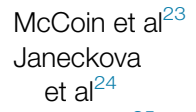 \\
\hline Urea cycle disorders & $\begin{array}{l}\text { Argininosuccinic acid } \\
\text { lyase (AL) } \\
\text { deficiency, } \\
\text { Argininemia, } \\
\text { ornithine } \\
\text { transcarbamylase } \\
\text { deficiency }\end{array}$ & Miller et $\mathrm{al}^{25}$ \\
\hline LSD & $\begin{array}{l}\text { Niemann-Pick C1, } \\
\text { Fabry, Pompe, } \\
\text { Gauche, Krabbe. } \\
\text { MPS (all types) }\end{array}$ & $\begin{array}{l}\text { Matern et } \mathrm{al}^{19} \\
\text { Fan et } \mathrm{al}^{26}\end{array}$ \\
\hline Organic acidemias & $\begin{array}{l}\text { MMA, PA, glutaric } \\
\text { aciduria type } 1, \\
\text { isovaleric acidemia }\end{array}$ & $\begin{array}{l}\text { Wikoff et } \mathrm{al}^{27} \\
\text { Miller et } \mathrm{al}^{25}\end{array}$ \\
\hline X-ALD & & Vogel et $\mathrm{al}^{20}$ \\
\hline $\begin{array}{l}\text { Mitochondrial } \\
\text { disorders }\end{array}$ & Leigh syndrome & $\begin{array}{l}\text { Thompson } \\
\text { et al }^{28} \\
\text { Barshop }^{29}\end{array}$ \\
\hline $\begin{array}{l}\text { Disorders of } \\
\text { cholesterol } \\
\text { biosynthesis }\end{array}$ & $\begin{array}{l}\text { CTX, (SLOS), } \\
\text { Sitosterolemia }\end{array}$ & $\begin{array}{l}\text { Vaz et al } \\
\text { Oostendorp } \\
\text { et } \mathrm{al}^{30}\end{array}$ \\
\hline Barth syndrome & & Sandlers et $\mathrm{al}^{31}$ \\
\hline $\begin{array}{l}\text { 3-methylcrotonyl-CoA } \\
\text { carboxylase }\end{array}$ & & Miller et $\mathrm{al}^{25}$ \\
\hline $\begin{array}{l}\text { 3-OH-3methylglutaryl } \\
\text { (HMG) CoA lyase } \\
\text { deficiency }\end{array}$ & & Miller et a ${ }^{25}$ \\
\hline $\begin{array}{l}\text { Guanidinoacetate } \\
\text { methyltransferase } \\
\text { (GAMT) deficiency }\end{array}$ & & Miller et al ${ }^{25}$ \\
\hline $\begin{array}{l}\text { Lysinuric protein } \\
\text { intolerance }\end{array}$ & & Miller et $\mathrm{al}^{25}$ \\
\hline
\end{tabular}

Abbreviations: CPT2, carnitine palmitoyltransferase 2 deficiency: $C T X$, cerebrotendinous xanthomatosis; $F A O D$, fatty acid oxidation disorders; $L C H A D$, long-chain 3-hydroxyacyl-CoA dehydrogenase; $L S D$, lysosomal storage diseases; $M C A D$, medium chain acyl CoA dehydrogenase deficiency; MMA, methylmalonic acidemia; MSUD, maple syrup urine disease; $P A$, propionic acidemia; $P K U$, phenylketonuria; SLOS, Smith-Lemli Opitz syndrome; VLCAD, very long-chain acyl-COA dehydrogenase deficiency.

that due to $\beta$-oxidation defect, long-chain fatty acids (LCFA) are rerouted to complex lipid biosynthetic pathways. These findings represent new insight in disease pathophysiology and may provide additional diagnostic biomarkers even for mild phenotypes.

Metabolic abnormalities in Barth syndrome have been investigated with the untargeted approach and provided new insight into the pathogenesis of the disease. Sandlers et al combined ${ }^{1} \mathrm{H}$ NMR 
and LC-MS/MS platforms to study biochemical abnormalities in the plasma of Barth syndrome patients. ${ }^{31}$ A supervised multivariate analysis (OPLSDA) of all metabolites detected in MS assay showed a clear distinction between Barth syndrome patients and controls. Statistically significant different metabolites identified in the multivariate data analysis of the NMR metabolomics data and those identified in the MS analysis were used in the pathway enrichment analysis that validated previously known plasma markers and associated new, broad metabolic dysregulations with the etiology of this multisystem disease. The study also revealed new therapeutic targets for further exploration in Barth Syndrome.

Miller et al. performed a retrospective diagnosis of 190 plasma samples with 120 confirmed IEM cases. The study applied an untargeted workflow based on an analysis by 3 separate MS platforms in parallel. ${ }^{25}$ Despite high intra-assay precision for most metabolites and the potential to cover multiple metabolites under one single test, the study yielded a few false-negative outcomes. Specifically, it failed to identify guanidinoacetate which led to failure in guanidinoacetate methyltransferase deficiency detection. In addition, long-chain 3-hydroxy acylcarnitines which are diagnostic for long-chain 3-hydroxyacyl-CoA dehydrogenase deficiency and trifunctional protein deficiency were missing from the detected metabolites. Metabolomic investigations also included 70 undiagnosed patients' samples however, obtained data did not facilitate correlation to clinical findings. Only two undiagnosed cases were uncovered as female heterozygous ornithine transcarbamylase deficiency and TMLHE deficiency (disorder of carnitine biosynthesis). Two additional cases were preliminarily identified as sarcosinemia.

\section{APPLICATIONS OF TARGETED METABOLOMICS IN IEM STUDIES}

The targeted metabolic approach is a common practice in biochemical genetics laboratories for biochemical diagnosis and monitoring IEMs such as aminoacidopathies, organic acidurias, and fatty acid oxidation disorders. For example, GC-MS is a gold standard platform for routine urinary organic acids analysis. Organic acids are extracted by acidic liquidliquid extraction and analyzed as methoxyamine/ trimethylsilyl derivatives. The deri-vatization step allows formation of more volatile compounds and creation of specific and recognizable fragmentation patterns of diagnostic metabolites.

Historically, analysis of plasma and urine amino acids has been performed by ion exchange chromatography followed by postcolumn derivatization and UV detection. With tandem MS and NBS test development, a small set of diagnostically significant amino acids are analyzed in dried blood spots with flow injection analysis (FIA) tandem MS. This extremely high-throughput and low-cost approach does not involve chromatographic separation. Furthermore, derivatization with hydrochloric acid/n-butanol provides high sensitivity and specificity to the assay and allows simultaneous analysis of amino acids and acylcarnitines. Despite the enormous diagnostic significance, FIA-MS/MS is a first-tier screening method and does not provide resolution of isomeric and isobaric metabolites. To achieve higher specificity, accuracy, and precision, a variety of GC-MS and LC-MS/MS methods have been developed. ${ }^{32,33}$

Different groups demonstrated the application of targeted metabolomics as a discovery tool for IEMs (Table II). Janeckova et al validated known diagnostic biomarkers in various metabolic disorders through a combination of chemometrics and quantitative targeted metabolomics. $^{24}$ The study analyzed 163 plasma metabolites and applied unsupervised statistical analysis to successfully detect 34 IEM patients. Fan et al explored a targeted metabolomics approach to test the hypothesis that specific plasma sphingolipids can serve as outcome measures in clinical trials for Niemann-Pick C1 (NPC1) disease therapies. ${ }^{26}$ First, blood and cerebrospinal fluid in animal models were analyzed and then the analysis was extended to 56 NPC1 and matching control human subjects. The human study found alterations in multiple plasma sphingolipid species, while aberrant sphingolipid metabolism is a known metabolic hallmark of NPC1 disease. The study then identified specific sphingolipids as potential biomarkers in plasma and cerebrospinal fluid in monitoring longitudinal miglustat intervention. Alterations in the discovered sphingolipids during preand postintervention periods suggest that these biomarkers may provide useful endpoints for monitoring the efficacy of drug candidates during clinical trials.

Diet represents a significant factor that affects the metabolome even in healthy individuals. IEM management often includes nutritional limitations and supplements. Medical foods for IEMs fall in few categories: foods that do not include "offending" amino acid, protein-restricted foods and foods that include some amino acids or vitamins, or other components used to replace essential nutrients or to enhance enzyme activity.

Although targeted relevant analysis is already in use to monitor nutritional management in many IEMs, the overall long-term impact of dietary restrictions is not clear. For example, in PKU patients only plasma phenylalanine to tyrosine ratio and phenylalanine level 
are routinely monitored with no consideration of overall metabolic profile.

Mutze et al. investigated the influence of the long-term PKU diet on fatty acid metabolism. ${ }^{22}$ The targeted metabolic study found no functional influence on unsaturated fatty acid metabolism however, it demonstrated that long-term dietary fatty acid restriction influences mitochondrial beta-oxidation intermediates. Karam et al ${ }^{34}$ analyzed cardiovascular disease biomarker levels in a group of patients with various IEMs maintained on protein-restricted diet for a number of years. Plasma cholesterol, triglycerides, lipoproteins, and total homocysteine (a thrombotic factor) were analyzed in a cohort of patients diagnosed with PKU, tyrosinemia type I, urea cycle disorders, branched chain organic academia, and healthy controls. No significant differences were found in cardiovascular disease risk markers between IEM patients and the control group, possibly due to decreased saturated fat and increased fiber in IEM patients' diet.

Despite a few published targeted studies on medical foods effects on metabolism, scientific and clinical communities are still missing comprehension of the complex network of interactions between medical nutrients and metabolism at global level. According to Camp et al, there is "gap in knowledge regarding the safety and utility of nutritional interventions for management of IEM" ${ }^{35}$ and there is a need for more studies on "impact of nutritional interventions on health outcomes and on the psychosocial issues identified by patients and their families."

\section{APPLICATION OF METABOLOMICS IN MITOCHONDRIAL DISORDERS}

Mitochondrial diseases are a group of very complex inherited disorders that can result from abnormalities in the mitochondrial and nuclear genomes. ${ }^{36}$ Nuclear DNA abnormalities can be inherited in an autosomal recessive or autosomal dominant manner, while mitochondrial DNA defects are transmitted through maternal inheritance. These are multisystem disorders with a highly variable clinical presentation and no specific and effective therapies. ${ }^{37}$ Patient management includes supportive treatments to maximize life quality and to modulate disease progression. Due to genotypic and phenotypic heterogeneities and small patient cohorts, there are only few metabolomics studies in mitochondrial disorders. Thompson et al investigated the metabolic presentation of a genetically homogenous, French Canadian variant of Leigh syndrome in 9 patients. ${ }^{28}$ This targeted metabolomics experimental design encompassed clinically established assays as well as targeted nonclinical GC-MS and LC-MS methods. The study resulted in 45 distinctive markers that provide possible therapeutic targets.
Retrospective analysis of urine organic acids from 3646 randomly selected samples was performed by Barshop, who found that the urinary lactate level is not a good discriminator of mitochondrial disease patients, but did find a correlation of fumarate and malate levels to the patients' diagnosis. ${ }^{29}$ Reinecke et al extended urinary organic acids analysis and performed an untargeted GC-MS study with 39 selected samples from children diagnosed with respiratory chain deficiencies and matching controls. ${ }^{38}$ Vigorous statistical analysis did not reveal unique diagnostic markers, although the study provided some global metabolic perturbations in urinary profiles as a consequence of the respiratory chain deficiencies.

\section{LIPIDOMICS STUDIES IN IEMS}

The lipidome refers to the organic solvent-soluble components of the metabolome; thus, it is recommended that these compounds be analyzed separately from water-soluble metabolites. In contrast to the metabolome, the lipidome can also be performed in a shotgun manner. This analytical approach allows the separation of lipids from other biological sample components by extraction and analysis of crude extracts directly by MS. ${ }^{39}$ Similar to metabolomic approaches, lipid analysis can be performed in either a global or targeted manner. Abnormal lipid metabolism ${ }^{40}$ including cholesterol biosynthesis pathways ${ }^{41}$ is associated with a rare but rapidly expanding group of inherited metabolic disorders. Recent developments in highthroughput assays for diagnostic markers and new treatment options led to pilot NBS programs for disorders of lipid metabolism in several states ${ }^{14}$ however, there is a growing demand for tools to assess and monitor treatment efficacy and safety. Application of global lipidomics for this group of inherited disorders provides such a tool and facilitates the development of new therapies. Byeon et al found variation in plasma and urinary lipids in Fabry disease patients under enzyme replacement therapy. ${ }^{42}$ The nontargeted nanoflow LC electrospray-ionization tandem MS (nLC-ESI-MS/ MS) approach led to the identification of 129 plasma and 111 urinary lipids. Based on the first nontargeted phase, authors selected 29 plasma and 23 urinary statistically significant species for targeted quantitative analysis to identify lipid alterations under enzyme replacement therapy.

Oostendorp et al. demonstrated a targeted application of $1 \mathrm{H}$ NMR and 2D-NMR to identify and quantify abnormal lipids in patients with a known inborn error of cholesterol biosynthesis. Based on plasma profiling of a predefined set of 9 lipids, the study successfully identified patients with Smith-Lemli Opitz syndrome, 
cerebrotendinous xanthomatosis (CTX), and sitosterolemia. ${ }^{30}$ The clinical picture of CTX is extremely heterogeneous and patients' responses to therapy can be different. Prognostic markers of cholesterol metabolism before and after chenodeoxycholic acid (CDCA) treatment were studied by Mignarri et al in 19 CTX patients by targeted GC-MS analysis. ${ }^{43}$ Although the study did not reveal biomarkers that can distinguish CTX-stable patients and those with neurologic progression, it found that CDCA treatment normalized overall abnormal sterols, with the exception of $7 \alpha$-hydroxy-4-cholesten-3-one ( $7 \alpha \mathrm{C} 4)$. In addition, levels of plant sterols were proposed to be monitored under CDCA therapy; especially lathosterol that may assist the clinician in deciding whether to add a statin to CDCA therapy.

\section{THE FUTURE PERSPECTIVE: METABOLOMICS IN LABORATORY MEDICINE}

Targeted metabolomics is already in current use and is widely implemented to achieve timely diagnoses and monitoring nutritional management in many IEMs. These assays are highly regulated and meet CLIA quality standards under nonwaived, laboratorydeveloped tests. Applications of untargeted metabolomics in the clinical laboratory are still in infancy however, it is expected to dramatically change the scope and utility of the clinical laboratory playing a significant role in patient management. Especially, global untargeted studies aim in defining metabolic set points as a long-term monitoring tool for nutritional interventions in many IEMs and enzyme replacement therapies in lysosomal storage disorders. It is also expected to reveal the variation in an individual's response to the therapy based on the collected data. Mitochondrial disorders group also will benefit from untargeted metabolomic studies, as these disorders suffer from a lack of diagnostic markers, and generation of large metabolic data sets offers a discovery tool for better diagnosis and identification of potential therapeutic targets.

Although incorporation of global metabolomics in clinical laboratories allows the detection of a wide range of metabolites in a single test and opens up new prospectives in precision and personalized medicine, the translation of large-scale untargeted metabolomics from the bench to the bedside is facing some challenges.

To deliver quality results, standardization and harmonization of many aspects of the metabolomics workflow are mandatory. Nonstandardized experimental designs, sample collection, storage, and pretreatment could have negative impact on the tests outcomes. Experimental designs should be planned carefully to minimize bias: age, gender, ethnicity, and basic diet need to be considered. Appropriate sample sizes are necessary to obtain the statistical power of the results. Since the human metabolome is very dynamic and affected by environmental factors, including nutrition and gut microflora products, criteria for sample rejection should be established and documented by the laboratory.

Moving to the analytical phase, there are no standard sample preparation methods and standard chromatography tools. Sample preparation is an important starting point of any analytical process. Most of the metabolomic workflows prefer nonselective sample preparation such as dilution or protein precipitation methods over more selective procedures like solid phase or liquid-liquid extractions. The sample preparation step is often minimized to increase analysis throughput and compatibility to automation however, matrix effect and ion suppression caused by proteins and phospholipids, especially in blood, serum, or plasma samples can decrease the sensitivity and selectivity of the analysis masking biological variation. In NMR, macromolecules and inorganic salts from the biological matrix can cause problems with baseline distortion and variation in the chemical shifts. In LC-MS/MS, metabolites co-eluting with matrix components will have different ionization efficiency and/or adduct formation which will affect their quantitative signals. A proper choice of the sample preparation will increase selectivity and may reduce the false-positive and false-negative discovery rate, thereby increasing the validity of any metabolomics investigation.

Most of the LC-MS/MS studies reviewed here were limited to reverse phase (RP) chromatography with various $\mathrm{C} 18$-bonded silica stationary phases. This type of analytical approach is robust, highly sensitive, and reproducible however, it introduces bias toward certain classes of analytes, as many clinically relevant metabolites are water soluble and thus are not retained on RP. To enhance the resolution of highly polar metabolites, hydrophilic interaction LC (HILIC) is recommended $^{44,45}$ and was demonstrated as an effective choice in urine metabolomics. ${ }^{46,47}$ Although the experimental setup of two column analyses requires later data fusion from two analyses, combination of RP and HILIC allows a broad metabolome coverage. Alternatively, 2-dimensional chromatography system can be a choice of the analytical platform. In this case although, due to differences in equilibration times and mobile phases incompatibility between RP and HILIC stationary phases, only RP columns should be employed for 2-dimensional chromatography system. ${ }^{48}$

Interlaboratory precision in metabolomics is a critical step toward standardization. There is a gap in 
standard and certified reference material availability. Uniform internal standards must be established and periodically validated in a similar manner as it is done in NBS laboratories. For many clinically relevant metabolites, there are no commercially available stable isotope-labeled standards, and it is a common practice in biochemical genetic laboratories to use single-point calibration for some targeted tests such as amino acids or acylcarnitines. Although single-point calibration and FIA-MS/MS significantly increase throughput, they are subject to ion suppression and matrix effects.

Siskos et al reported a quantitative targeted metabolomics reproducibility study ${ }^{49}$ which involved 6 laboratories performing the analysis of identical plasma and serum samples through different instrumentation but a common protocol for the analysis of 189 metabolites via LC-MS/MS or FIA-MS/MS. Quantitative LC-MS/MS analysis was achieved by 7-point calibration curves with isotope-labeled internal standards and levels of 146 metabolites under study were obtained by single-point calibration FIA-MS/MS analysis. To achieve high interlaboratory reproducibility for FIA-MS/MS, the authors used data normalization. Overall, this work demonstrated high reproducibility across participating laboratories for the established protocol and normalization approach for single-point calibration methods that can be integrated in large-scale metabolomics studies.

The interlaboratory performance evaluation of untargeted metabolomics studies however, does not look very optimistic. The Metabolomics Research Group of the Association of Biomolecular Resource Facilities reported a study that involved 14 participating laboratories with different analytical platforms. ${ }^{50}$ The goal of the investigation was to replicate an initial quantitative metabolomics discovery experiment that was performed by core facility. The study concluded that application of more than one analytical platform provides broad metabolome coverage and increases chances for identification of unknowns. At the same time, some laboratories reported a change in the relative concentration of metabolites, but in opposite direction. In addition, quantitation of spiked metabolites with high endogenous levels was not as accurate as those that had lower endogenous levels. The biggest challenge, however, reported in the study was unknowns identification.

Several algorithms and workflows have been developed to reduce within-experiment analytical variation ${ }^{51-53}$ however, there is no uniform approach in data handling, processing, or metabolite identification tools which are critical components for generating scientifically meaningful results. The Metabolomic Standard
Initiative is a group of highly qualified scientists who recently published recommended reporting standards for untargeted metabolomics studies. Published guidelines refer to all aspects of untargeted metabolomics workflow for NMR and MS methods "to develop consistent and appropriate descriptors to support the dissemination and reuse of metabolomic data." ${ }^{54}$ The outcomes from the global discovery phase provide metabolites associated with the biological status. At this stage, statistically discriminating metabolites need to be identified and validated. Despite accurate mass and fragment ions pattern generated by MS, it is still a very challenging and time-demanding task. To facilitate metabolite identification, several databases are available based on MS and NMR acquisitions. ${ }^{55-58}$ In the next step, the identified potential biomarkers must be validated analytically and biologically through additional cross studies. Currently, there is a lack of a coherent pipeline connecting the biomarker discovery phase with the biomarker validation phase.

Most of the untargeted studies demonstrate the ability of metabolite profiling to distinguish between disease and healthy control cohorts however, only few studies follow up to replicate and validate discovery outcomes in accordance with clinical performance standards. It is crucial to understand that biomarker detection and identification only represent the starting point in the translation from discovery phase to clinical diagnostics.

In most metabolomics studies, a list of "putative biomarkers" is selected based on univariate or multivariate statistical approaches that capture data variance. Multivariate techniques are potentially more powerful, since univariate models could overestimate the significance of certain variables, because correlation between variables is not taken into account. In other words, treating the data with univariate analysis does not always provide a good predictive model particularly for IEMs. For many IEMs, a metabolic pattern rather than a single biomarker is diagnostic. For example, urea cycle defects such as carbamylphosphate synthetase deficiency or ornithine transcarbamylase deficiency are accompanied by low plasma citrulline levels. However, low plasma citrulline levels will be also observed secondary to mitochondrial dysfunction, an extended period of reduced protein nutrition, or a disease of the gastrointestinal tract, where citrulline is synthesized. In the case of low plasma citrulline, glutamine levels should be examined to exclude hyperammonemia. The levels of some essential amino acids will also be evaluated to exclude malnutrition and sample hemolysis.

The selection of highly ranked metabolites by multivariate statistics most of the time is also supported by regression analysis, $P$ values, or variable importance in projection scores (VIP). While these algorithms are 
very important for understanding of disease mechanism, they do not provide information on biomarker performance by a clinical meaning. Clinical biomarker performance evaluation has to address the sensitivity and specificity at the specific cut-offs. For this purpose, a receiver-operating characteristic (ROC) curve is widely applied. ${ }^{59}$ ROC describes the relationship between the sensitivity and specificity of any diagnostic test at various decision thresholds. The area under the curve reflects the ability of the test to discriminate between true-positive and true-negative results.

Additional challenge is that IEMs are a group of relatively rare disorders thus, studies usually include small cohorts of affected patients. In biomarkers discovery, a small sample size will not provide enough information to build a predictive model. To allow unbiased estimates of model performance, crossvalidation methods are recommended. This approach uses a subsampling of the data to estimate the predictive power of the small cohort model in a large population. $^{60,61}$

For example, Lee et al designed and performed a study to validate a panel of nephrotic syndrome urinary markers. ${ }^{62}$ The experimental design of the study encompasses untargeted metabolomics approach followed by the validation of highly ranked metabolites in constructed panels. Multistage statistical tools were applied to select biomarker panels from a discovery samples set and to validate the selected biomarkers through an independent set of urine sample analyses. The selectivity and sensitivity of putative biomarkers were assessed through the construction of ROC curves and the calculation of area under the curve.

Another significant gap that needs to be addressed for future large-scale metabolomic clinical applications is the development of external quality assurance programs. Such programs will improve the robustness and validity of clinical data and guarantee the comparability of results between different laboratories. In 2016, the National Institute of Standards and Technology (NIST) launched a pilot quality assurance program, "qMet", to study and address quality control deficiencies across metabolomic studies with NMR, GC-MS, and LC-MS (https://www.nist.gov/programsprojects/qmet-quality-assurance-program-meta

bolomics). This effort, combined with the regulatory agencies' collaboration, will expedite metabolomics incorporation in clinical practices through the development of new recommendations, protocols, and policies.

In summary, the field of targeted metabolomics with respect to diagnostic biomarkers detection in IEMs will continue to be important in future clinical settings. Global, untargeted metabolomics holds the promise of better IEM phenotypes characterization, potentially providing the clinician the ultimate information for personalized care. In combination with genomics, large-scale untargeted metabolomics is expected to reveal genotype-phenotype correlations and the overall effect of drugs and dietary interventions however, for the successful translation of the global metabolomics from bench to the bedside, some gaps need to be bridged and quality control challenges need to be addressed.

\section{ACKNOWLEDGMENTS}

Conflicts of Interest: All the authors have read the journal's authorship agreement and the journal's policy on disclosure of potential conflict of interest and have nothing to declare.

The authors would like to thank Ms. Erica Fatica for assistance in manuscript editing.

\section{REFERENCES}

1. Nicholson JK, Lindon JC. Systems biology: Metabonomics. Nature 2008;455:1054-6.

2. Horning EC. Use of combined gas-liquid chromatography and mass spectrometry for clinical problems. Clin Chem 1968;14:777.

3. Behar KL, Denhollander JA, Stromski ME, et al. High resolution H-1 nuclear magnetic resonance study of cerebral hypoxia in vivo. Proc Natl Acad Sci U S A 1983;80:4945-8.

4. Oliver SG, Winson MK, Kell DB, Baganz F. Systematic functional analysis of the yeast genome. Trends Biotechnol 1998;16:373-8.

5. Nicholson JK, Lindon JC, Holmes E. "Metabonomics": understanding the metabolic responses of living systems to pathophysiological stimuli via multivariate statistical analysis of biological NMR spectroscopic data. Xenobiotica 1999;29: 1181-9.

6. Clendinen CS, Lee-McMullen B, Williams CM, et al. 13C NMR metabolomics: applications at natural abundance. Anal Chem 2014;86:9242-50.

7. Aruni DeSilva M, Shanaiah N, Gowda GAN, Rosa-Pérez K, Hanson BA, Raftery D. Application of ${ }^{31} \mathrm{P}$ NMR spectroscopy and chemical derivatization for metabolite profiling of lipophilic compounds in human serum. Magn Reson Chem 2009;47:S74-80.

8. Karaman I. Preprocessing and pretreatment of metabolomics data for statistical analysis. Adv Exp Med Biol 2017;965:145-61.

9. Ren S, Hinzman AA, Kang EL, Szczesniak-Long RD, Lu J. Computational and statistical analysis of metabolomics data. Metabolomics 2015;11:1492-513.

10. Kankainen M, Gopalacharyulu P, Holm L, Oresic M. MPEAmetabolite pathway enrichment analysis. Bioinformatics 2011; 27:1878-9.

11. Chagoyen M, Pazos F. Tools for the functional interpretation of metabolomic experiments. Brief Bioinform 2013;14:737-44.

12. Mak CM, Lee HC, Chan AY, Lam CW. Inborn errors of metabolism and expanded newborn screening: review and update. Crit Rev Clin Lab Sci 2013;50:142-62.

13. Vernon H. Inborn errors of metabolism advances in diagnosis and therapy. JAMA Pediatr 2015;169:778-82.

14. Wilcken B, Wiley V. Newborn screening. Pathology 2008;40: 104-15.

15. Millington DS, Kodo N, Norwood DL, Roe CR. Tandem mass spectrometry: a new method for acylcarnitine profiling with 
potential for neonatal screening for inborn errors of metabolism. J Inherit Metab Dis 1990;13:321-4.

16. Chace DH, Hillman SL, Millington DS, Kahler SG, Roe CR, Naylor EW. Rapid diagnosis of maple syrup urine disease in blood spots from newborns by tandem mass spectrometry. Clin Chem 1995;41:62-8.

17. Rinaldo P, Cowan TM, Matern D. Acylcarnitine profile analysis. Genet Med 2008;10:151-6.

18. Chace DH, Kalas TA, Naylor EW. Use of tandem mass spectrometry for multianalyte screening of dried blood specimens from newborns. Clin Chem 2003;49:1797-817.

19. Matern D, Gavrilov D, Oglesbee D, Raymond K, Rinaldo P, Tortorelli S. Newborn screening for lysosomal storage disorders. Semin Perinatol 2015;39:206-16.

20. Vogel BH, Bradley SE, Adams DJ, et al. Newborn screening for X linked adrenoleukodystrophy in New York State: diagnostic protocol, surveillance protocol and treatment guidelines. Mol Genet Metab 2015;114:599-603.

21. Vaz FM, Bootsma AH, Kulik W, et al. A newborn screening method for cerebrotendinous xanthomatosis using bile alcohol glucuronides and metabolite ratios. J Lipid Res 2017;58:1002-7.

22. Mütze U, Beblo S, Kortz L, et al. Metabolomics of dietary fatty acid restriction in patients with phenylketonuria. PLoS One 2012; 7:e43021.

23. McCoin CS, Piccolo BD, Knotts TA. Unique plasma metabolomic signatures of individuals with inherited disorders of long-chain fatty acid oxidation. J Inherit Metab Dis 2016;39:399-408.

24. Janeckova H, Hron K, Wojtowicz P, et al. Targeted metabolomic analysis of plasma samples for the diagnosis of inherited metabolic disorders. J Chromatogr A 2012;1226:11-7.

25. Miller MJ, Kennedy AD, Eckhart AD, et al. Untargeted metabolomic analysis for the clinical screening of inborn errors of metabolism. J Inherit Metab Dis 2015;38:1029-39.

26. Fan M, Sidhu R, Fujiwara H, et al. Identification of Niemann-Pick C1 disease biomarkers through sphingolipid profiling. J Lipid Res 2013;54:2800-14.

27. Wikoff WR, Gangoiti JA, Barshop BA, Siuzdak G. Metabolomics identifies perturbations in human disorders of propionate metabolism. Clin Chem 2007;53:2169-76.

28. Thompson LJ, Strittmatter L, Tardif J. A metabolic signature of mitochondrial dysfunction revealed through a monogenic form of Leigh Syndrome. Cell Rep 2015;13:981-9.

29. Barshop BA. Metabolomic approaches to mitochondrial disease: correlation of urine organic acids. Mitochondrion 2004;4:521-7.

30. Oostendorp M, Engelke UF, Willemsen MA, Wevers RA. Diagnosing inborn errors of lipid metabolism with proton nuclear magnetic resonance spectroscopy. Clin Chem 2006;52:1395-405.

31. Sandlers Y, Mercier K, Pathmasiri W, et al. Metabolomics reveals new mechanisms for pathogenesis in Barth Syndrome and introduces novel roles for cardiolipin in cellular function. PLoS One 2016;11:e0151802.

32. Minkler PE, Stoll MS, Ingalls ST, Yang S, Kerner J, Hoppel CL. Quantification of carnitine and acylcarnitines in biological matrices by HPLC electrospray ionization mass spectrometry. Clin Chem 2008;54:1443-54.

33. Filee R, Schoos R, Boemer F. Evaluation of physiological amino acids profiling by tandem mass spectrometry. JIMD Rep 2014;13: 119-28.

34. Karam PE, Majdalani MN, Daher RT, Barhoumi A, Yazbeck N. Cardiovascular disease biomarkers in patients with inborn errors of protein metabolism: a pilot study. J Hum Nutr Diet 2015;28: 344-9.

35. Camp KM, Lloyd-Puryear MA, Yao L, et al. Expanding research to provide an evidence base for nutritional interventions for the management of inborn errors of metabolism. Mol Genet Metab 2013;109:319-28.

36. Lightowlers RN, Taylor RW, Turnbull DM. Mutations causing mitochondrial disease: what is new and what challenges remain. Science 2015;349:1494-9.

37. El-Hattaba AW, Scaglia F. Mitochondrial cytopathies. Cell Calcium 2016;60:199-206.

38. Reinecke CJ, Koekemoer G, van der Westhuizen FH, et al. Metabolomics of urinary organic acids in respiratory chain deficiencies in children. Metabolomics 2012;8:264-83.

39. Han X, Gross RW. Shotgun lipidomics: electrospray ionization mass spectrometric analysis and quantitation of cellular lipidomes directly from crude extracts of biological samples. Mass Spectrom Rev 2005;24:367-412.

40. Garcia-Cazorla À, Mochel F, Lamari F, Saudubray JM. The clinical spectrum of inherited diseases involved in the synthesis and remodeling of complex lipids. A tentative overview. J Inherit Metab Dis 2015;38:19-40.

41. Kelley RI, Herman GE. Inborn errors of sterol biosynthesis. Annu Rev Genomics Hum Genet 2001;2:299-341.

42. Byeon SK, Kim JY, Lee JS, Moon MH. Variations in plasma and urinary lipids in response to enzyme replacement therapy for Fabry disease patients by nanoflow UPLC-ESI-MS/MS. Anal Bioanal Chem 2016;408:2265-74.

43. Mignarri A, Magni A, Del Puppo M, et al. Evaluation of cholesterol metabolism in cerebrotendinous xanthomatosis. J Inherit Metab Dis 2016;39:75-83.

44. Vorkas PA, Isaac G, Anwar MA, Davies AH, Want EJ, Nicholson JK, Holmes E. Untargeted UPLC-MS profiling pipeline to expand tissue metabolome coverage: application to cardiovascular disease. Anal Chem 2015;87:4184-93.

45. Ivanisevic J, Zhu ZJ, Plate L, et al. Toward 'omic scale metabolite profiling: a dual separation-mass spectrometry approach for coverage of lipid and central carbon metabolism. Anal Chem 2013;85:6876-84.

46. Spagou K, Wilson ID, Masson P, et al. HILIC-UPLC-MS for exploratory urinary metabolic profiling in toxicological studies. Anal Chem 2011;83:382-90.

47. Trevedi DK, Iles RK. HILIC-MS-based shotgun metabolomic profiling of maternal urine at 9-23 weeks of gestation - establishing the baseline changes in the maternal metabolome. Biomed Chromatogr 2015;29:240-5.

48. Ortmayr K, Causon TJ, Hann S, Koellensperger G. Increasing selectivity and coverage in LC-MS based metabolome analysis. Trends Anal Chem 2016;82:358-66.

49. Siskos AP, Jain P, Römisch-Margl W, et al. Interlaboratory reproducibility of a targeted metabolomics platform for analysis of human serum and plasma. Anal Chem 2017;89:656-65.

50. Cheema AK, Asara JM, Wang Y, Neubert TA, Tolstikov V, Turck CW. The ABRF metabolomics research group 2013 study: investigation of spiked compound differences in a human plasma matrix. J Biomol Tech 2015;26:83-9.

51. Kamleh MA, Ebbels TM, Spagou K, Masson P, Want EJ. Optimizing the use of quality control samples for signal drift correction in large-scale urine metabolic profiling studies. Anal Chem 2012;84:2670-7.

52. Kirwan JA, Broadhurst DI, Davidson RL, Viant MR. Characterising and correcting batch variation in an automated direct infusion mass spectrometry (DIMS) metabolomics workflow. Anal Bioanal Chem 2013;405:5147-57.

53. Brunius C, Shi L, Landberg R. Large-scale untargeted LC-MS metabolomics data correction using between-batch feature alignment and cluster-based within-batch signal intensity drift correction. Metabolomics 2016;12:173. 
54. Sumner LW, Amberg A, Barrett D, et al. Proposed minimum reporting standards for chemical analysis chemical analysis working group (CAWG) metabolomics standards initiative (MSI). Metabolomics 2007;3:211-21.

55. Tautenhahn R, Cho K, Uritboonthai W, Zhu Z, Patti GJ, Siuzdak G. An accelerated workflow for untargeted metabolomics using the METLIN database. Nat Biotechnol 2012;30:826-8.

56. Wang Y, Kora G, Bowen BP, Pan C. MIDAS: a database-searching algorithm for metabolite identification in metabolomics. Anal Chem 2014;86:9496-503.

57. Bingol K, Bruschweiler-Li L, Yu C, Somogyi A, Zhang F, Brüschweiler R. Metabolomics beyond spectroscopic databases: a combined MS/NMR strategy for the rapid identification of new metabolites in complex mixtures. Anal Chem 2015;87: 3864-70.
58. Zhou B, Wang J, Ressom HW. MetaboSearch: tool for mass-based metabolite identification using multiple databases. PLoS One 2012;7:e40096.

59. Obuchowski NA, Lieber ML, Wians FH. ROC curves in clinical chemistry: uses, misuses, and possible solutions. Clin Chem 2004;50:1118-25.

60. Xia J, Broadhurst DI, Wilson M, Wishart DS. Translational biomarker discovery in clinical metabolomics: an introductory tutorial. Metabolomics 2013;9:280-99.

61. Stone M. Cross-validatory choice and assessment of statistical predictions. J R Statist Soc B 1974;36:111-47.

62. Lee JU, Lee YH, Kim SY, et al. Systematic biomarker discovery and coordinative validation for different primary nephrotic syndromes using gas chromatography-mass spectrometry. J Chromatogr A 2016;1453:105-15. 